\title{
BurstCube: status and public alerts
}

Israel Martinez-Castellanos, ${ }^{m, p},{ }^{*}$ Isabella Brewer, ${ }^{a}$ Michael S. Briggs, ${ }^{b}$ Alessandro Bruno, ${ }^{c, d}$ Eric Burns, ${ }^{e}$ Regina Caputo, ${ }^{c}$ Brad Cenko, ${ }^{c}$ Georgia de Nolfo, ${ }^{c}$ Adam Goldstein, ${ }^{f}$ Sean Griffin, ${ }^{g}$ Sylvain Guiriec, ${ }^{h, c}$ Lorraine Hanlon, ${ }^{i}$ Dieter H. Hartmann, ${ }^{j}$ Boyan Hristov, ${ }^{b}$ Michelle Hui, ${ }^{k}$ Alyson Joens, ${ }^{h, c}$ Carolyn Kierans, ${ }^{c}$ Marc Kippen, ${ }^{l}$ Dan Kocevski, ${ }^{k}$ John Krizmanic, ${ }^{m, n}$ Sibasish Laha, ${ }^{m}$ Amy Lien, ${ }^{o}$ Sheila McBreen, ${ }^{i}$ Julie E. McEnery, ${ }^{c}$ J. Grant Mitchell, ${ }^{h, c}$ Lee Mitchell, ${ }^{q}$ David Morris, ${ }^{r}$ David Murphy, ${ }^{i}$ Pi Nuessle, ${ }^{h, c}$ Jeremy S. Perkins, ${ }^{c}$ Judith L. Racusin, ${ }^{c}$ Oliver Roberts, ${ }^{f}$ Peter Shawhan, ${ }^{p}$ Jacob R. Smith, ${ }^{m, n}$ Teresa Tatoli, ${ }^{c, d}$ Alexey Uliyanov, ${ }^{i}$ Sarah Walsh ${ }^{i}$ and Colleen A. Wilson-Hodge ${ }^{k}$

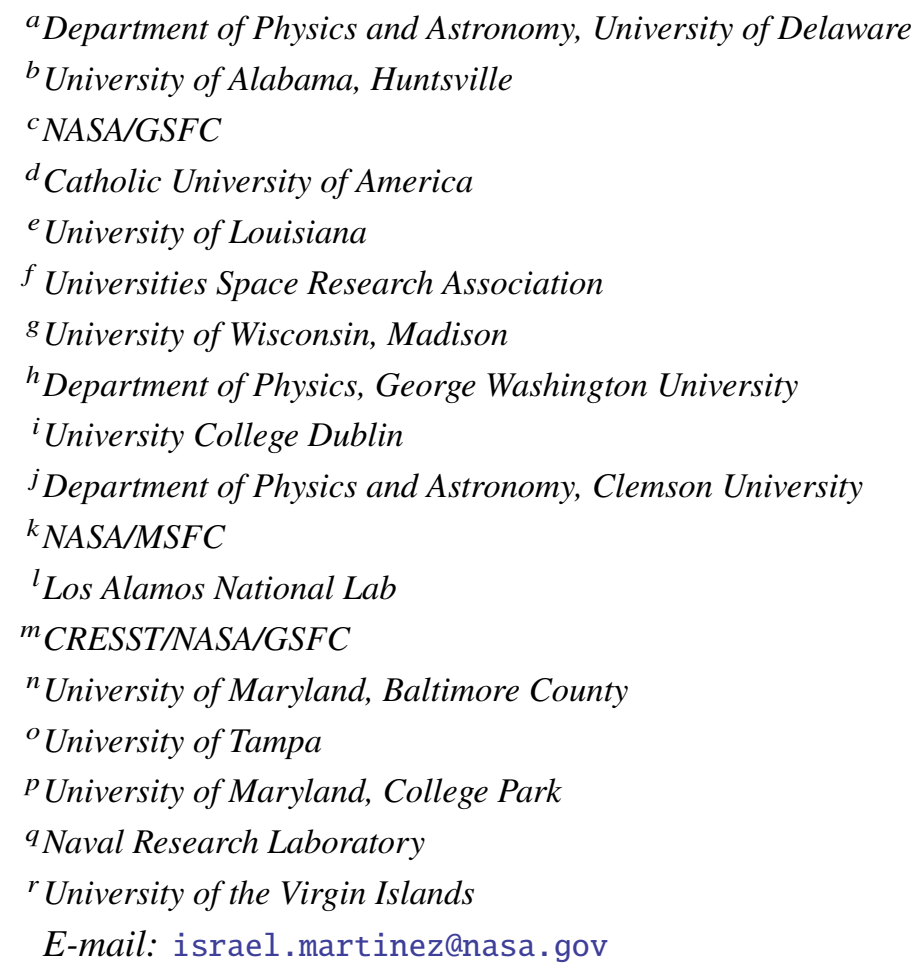

\footnotetext{
*Presenter
} 
BurstCube is a CubeSat Gamma-Ray Burst (GRB) detector expected to launch in 2022, significantly expanding sky coverage in the $50 \mathrm{keV}$ to $1 \mathrm{MeV}$ energy range and increasing the probability of detecting a gravitational wave counterpart. BurstCube will take advantage of the Tracking and Data Relay Satellite (TDRS) system in order to provide rapid public alerts and localization information, which has proven to be key to obtain valuable multi-messenger and multi-wavelength information. The BurstCube instrument is comprised of four Cesium Iodide scintillators coupled to arrays of Silicon Photomultipliers, therefore serving as a pathfinder for future missions utilizing this technology for space-based gamma-ray detection. In this presentation we will discuss the current status of the BurstCube instrument assembly, calibration and analysis pipelines. We will also describe the real-time alerts that will be sent out to the community throughout the duration of the mission. 


\section{Introduction}

The joint detection of GW170817, by the Laser Interferometer GW Observatory (LIGO), and GRB17087A, by the Fermi Gamma-Ray Burst Monitor (GBM), confirmed Binary Neutron Star mergers as progenitors of short Gamma-Ray Bursts (sGRBs) [1]. It represents a major milestone in multi-messenger astronomy, but no further coincident events have been observed. Since the current network of GRB observatories have approximately a 70\% sky coverage, a new mission can significantly increase the likelihood of a coincident detection and improve our chances of localizing the electromagnetic counterpart of a gravitational wave.

BurstCube is a $6 \mathrm{U}$ CubeSat $(1 \mathrm{U} \approx 10 \mathrm{~cm} \times 10 \mathrm{~cm} \times 10 \mathrm{~cm})$ sensitive to $50 \mathrm{keV}$ to $1 \mathrm{MeV}$ gammarays expected to launch in Fall 2022. It consists of four Cesium Iodide (CsI(Tl)) scintillators readout by an array of Silicon Photomultipliers (SiPMs). BurstCube will join the coordinated efforts of space and ground-based observatories to detect gravitational wave counterparts by providing rapid public alerts and localization information to the community.

\section{The BurstCube instrument}

The BurstCube instrument consists of 4 photon-counting detectors, each contained within approximately $1 \mathrm{U}$. The detectors are composed of a cesium iodide (CsI(Tl)) scintillator, $90 \mathrm{~mm}$ in diameter and $19 \mathrm{~mm}$ thick, optically coupled to an array of Silicons Photomultipliers (SiPMs), as shown in Figure 1c. The scintillators convert gamma rays into optical photons, which are then detected by the SiPMs and output as an electrical pulse. The energy of incident gamma-rays is proportional to the pulse height of the signal from the SiPM array. This combined with the count rate and the knowledge of the effective area (Figure 2a) allows for the reconstruction of the spectrum of the source.

The detectors are arranged in a pyramidal shape, each facing approximately $45^{\circ}$ from vertical (see Figure 1b). Since the acceptance decays as a function of the angle with respect to the detector's face normal (see Figure 2b), the relative rates detected on each detector are used to reconstruct the direction of a source. The attenuation by other spacecraft components and the mutual shadowing among the detectors also play a role while localizing a source, discussed in Section 3.

Overall the working principle is similar to that of Fermi-GBM. However, the use of SiPMs, as opposed to traditional photomultiplier vacuum tubes, allows for a compact design with low power consumption. BurstCube will complement other GRB observatories by increasing the field of view and up-time, and will also serve as a pathfinder for the use of SiPMs for the next generation of gamma-ray detectors in this energy range.

The BurstCube is currently undergoing assembly, test, and calibration. The four individual detectors are now fully built and they will be integrated as a single instrument through the rest of 2021. Preliminary tests shown in Figure 3 confirm an energy range from $<50 \mathrm{keV}$ to $>1 \mathrm{MeV}$, with a resolution of $\sim 30 \%$ at threshold and $\sim 10 \%$ in the hundreds $\mathrm{keV}$ regime.

The spacecraft bus is being assembled in parallel. Payload integration will follow the instrument calibration. Another set of comprehensive tests and calibration procedures will occur when the full spacecraft is assembled. BurstCube is currently scheduled to launch during in late 2022 (prior to 


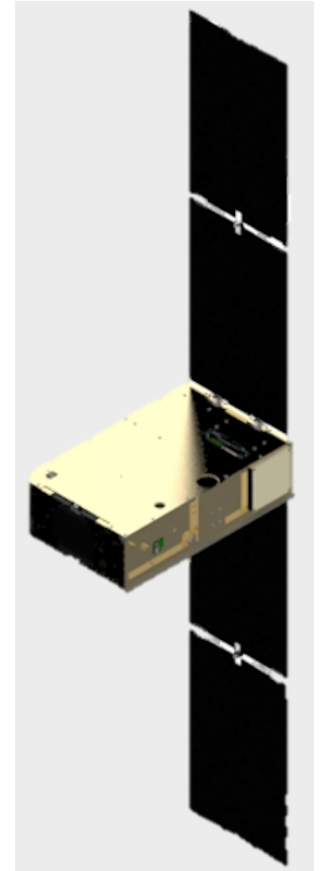

(a)

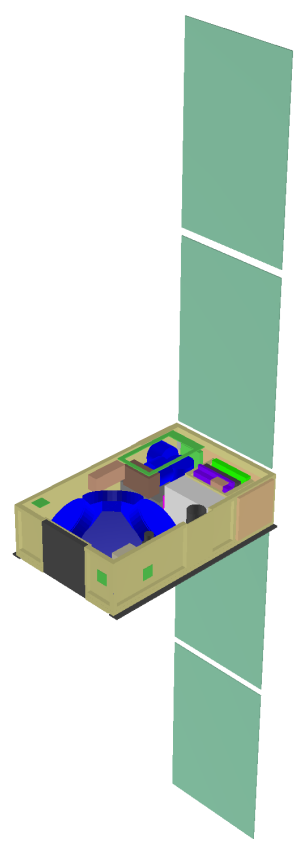

(b)

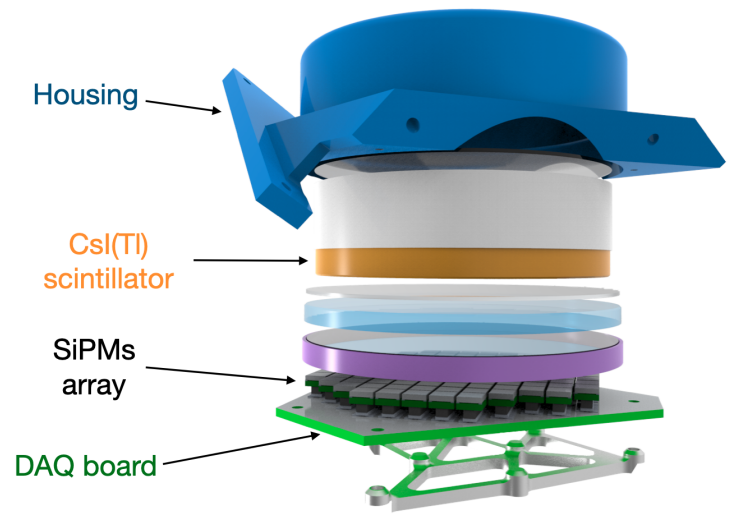

(c)

Figure 1: (a) A render of the BurstCube satellite with the solar panels deployed. (b) The mass model used for simulations. The instrument with four Single Quarter Detectors (SQDs) is shown in blue on the farthest side from the solar panels. (c) An expanded view of a single quarter detector. The main components are labeled. Other components include reflective materials, shock absorbing pads and layers to optically couple the scintillator to the SiPMs.

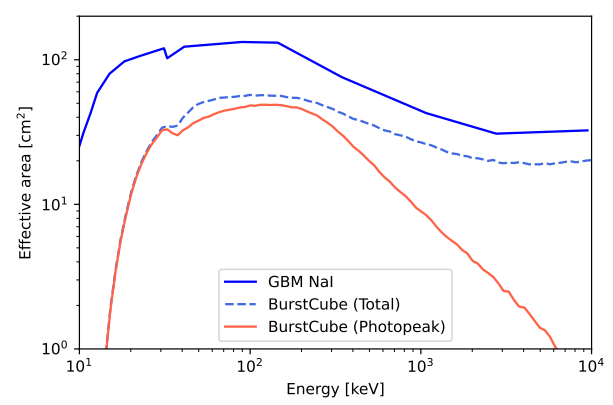

(a)

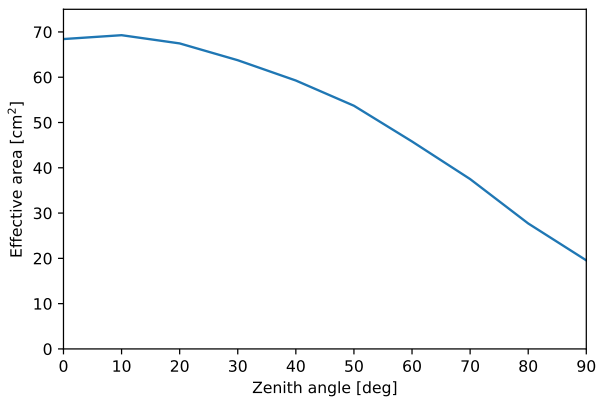

(b)

Figure 2: The effective area of a Single Quarter Detector (SQD). As a function of energy, in the normal direction (a) and as a function of the angle from the normal direction $100 \mathrm{keV}$ (b). The difference with respect to a GBM Nal detector effective area is mainly due to the physical area of their scintillator. 


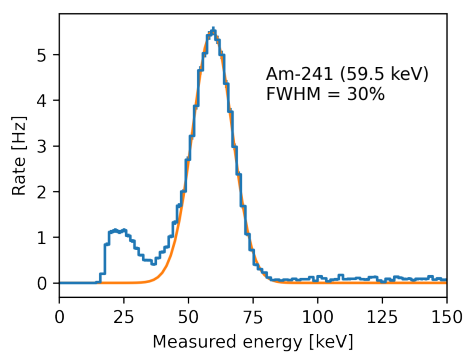

(a)

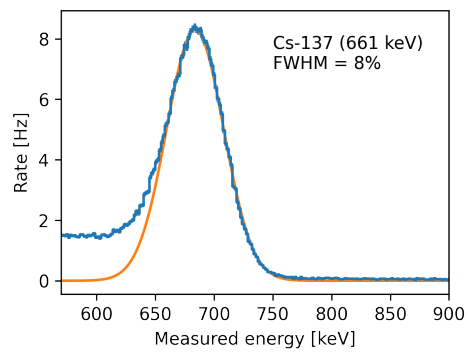

(b)

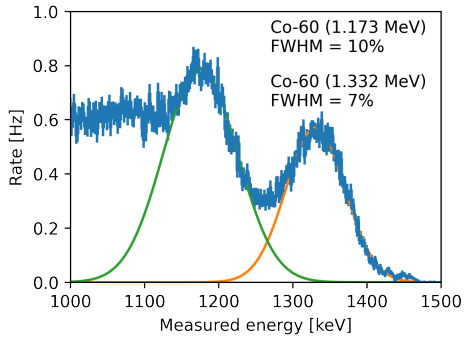

(c)

Figure 3: Measurements of the energy range $(<50 \mathrm{keV}$ to $>1 \mathrm{MeV})$ and resolution $(30 \%-10 \%)$ for the flight units, showing the fitted photopeaks for various radioactive sources ((a) Am-241, (b) Cs-137 and (c) Co-60).

COVID-19 the launch readiness date was Fall 2021), to be later deployed from the International Space Station.

\section{Simulations and analysis pipelines}

The main software package developed for BurstCube is called bc-tools ${ }^{1}$, an open-source Python library. It is designed do most of the end-to-end tasks expected from a count-based gamma-ray detector: data files processing, detector response generation, localization, source classification, burst duration estimation, binning, background estimation, spectral fitting and source injections. Some of these features are still under development. The code is detector agnostic and configurable, aiming to be of used for other similar CubeSats and BurstCube successors.

The bc-tools library builds upon two existing packages: MEGAlib [2] and the GBM Data Tools ${ }^{2}$. MEGAlib uses GEANT4 [3] to perform particle-by-particle Monte Carlo simulations. It takes a mass model as input, shown in Figure 1b, and computes the energy deposited onto active detectors (the $\mathrm{CsI}(\mathrm{Tl})$ scintillators for Burstcube). It therefore takes into account all reflections and absorption by other components of the spacecraft. Through a configuration file, the bc-tool library can apply additional detector effects in order to match the calibration results, such as energy resolution and bias. The resulting effective area, photon energy to measured energy dispersion and acceptance as a function of the incoming direction are encoded into detector matrices used for sky localization and spectral fitting.

GBM Data Tools is a Python API for the Fermi-GBM data. It allows for data reduction, binning, background estimation and spectral fitting. The goal of using GBM Data tools is two-fold: it avoids duplicating code to achieve the same functionality and facilitates backwards compatibility with GBM data products. BurstCube will also provide its data in common formats used by GBM.

Source localization is performed through a Poisson-based maximum likelihood method, using observed counts, estimated background, and detector response. A fiducial $1 \mathrm{~s}$ burst with a flux of $10 \mathrm{ph} \mathrm{cm}^{-2} \mathrm{~s}^{-1}$ (50-300 keV) occurring within $60^{\circ}$ from the instrument zenith can be localized to within a $15^{\circ}-30^{\circ}$ radius $(90 \%$ containment), as shown in Figure 4 . However, due to the axes of symmetry of the detector and other near acceptance degeneracies - e.g. downwards and upwards

${ }^{1}$ https://gitlab.com/burstcube/bc-tools

${ }^{2}$ https://fermi.gsfc.nasa.gov/ssc/data/analysis/rmfit/gbm_data_tools/gdt-docs/ 


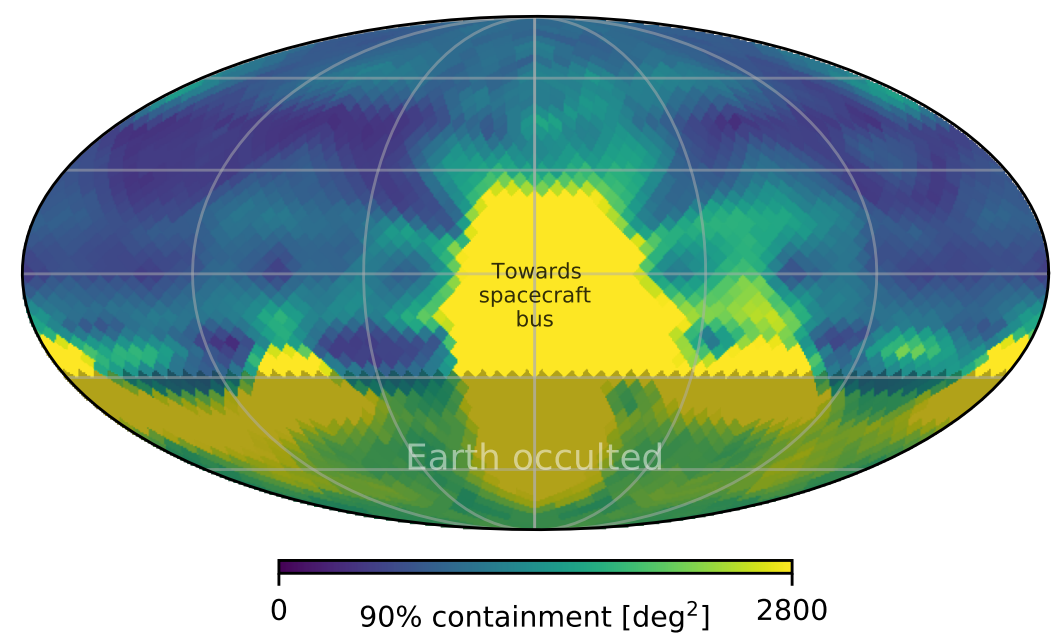

Figure 4: Expected localization resolution ( $90 \%$ containment) for a fiducial $1 \mathrm{~s}$ burst with a flux of $10 \mathrm{ph} \mathrm{cm}^{-2} \mathrm{~s}^{-1}(50-300 \mathrm{keV})$. In most directions this fiducial event would be localized to within a $<30^{\circ}$ radius. The localization constrain for sources being shadowed by the bulk of the spacecraft components is poor, except for the brightest events. Since most of the time BurstCube will be pointing towards zenith, the localization can be further constrained by discarding the portion of the localization contour occulted by the Earth. See also Figure 5.

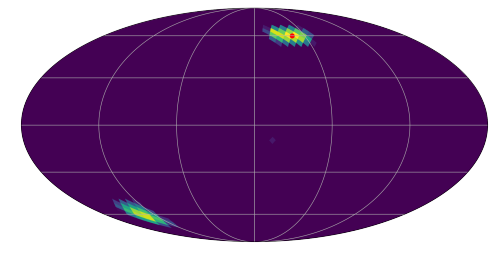

(a)

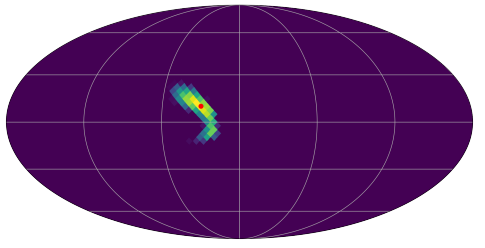

(b)

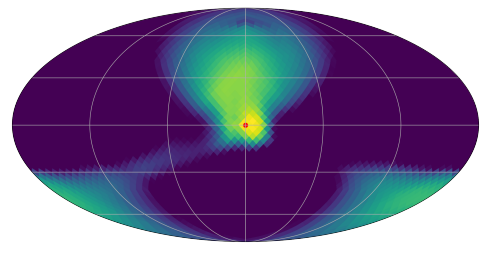

(c)

Figure 5: Mock maps for a fiducial $1 \mathrm{~s}$ burst with a $10 \mathrm{ph} \mathrm{cm}^{-2} \mathrm{~s}^{-1}$ flux. The red dot shows the real location of the injected source. (a) An example of the upward-downward degeneracy in the acceptance for some directions. The upwards direction will typically be in the Earth-occulted region and therefore ignored. (b) The localization uncertainty is not always well-represented by an ellipse. c An event coming through the bulk of the spacecraft bus. Attenuation by the passive components makes for a poor localization, except for the brightest events.

incoming directions - the localization contours are not always well described by an ellipse, as shown in Figure 5. This is one of the motivations to share localization information through sky maps.

\section{Expectation for public alerts}

BurstCube will be monitoring the sky with $\sim 90 \%$ uptime (shut down during the South Atlantic Anomaly (SAA) passage). Among other sources, it is expected to detect approximately $20 \mathrm{yr}^{-1}$ short GRBs during its 1-3 years of operation. All triggers and their localization will be publically available through the Gamma-ray Coordination Network (GCN) within 15 min of detection, most within $1 \mathrm{~min}$. 
In order to achieve a rapid alert dissemination BurstCube will transmit trigger information through the Tracking and Data Relay Satellite System (TDRSS), a new accomplishment for a CubeSat. TDRSS is able to relay data to the ground on demand within seconds. However, since BurstCube will only be within range of a TDRSS satellite $95 \%$ of the time there is the possibility of a $\sim 10$ min delay in some cases.

BurstCube will produce two types of data: pre-binned — Continuous Binned Data (CBD) and unbinned - Time-Tagged Events (TTE). Due to bandwidth constrains only CBD data will be downlinked in its totality, while TTE will only be available around events of interest. The TTE data will not be part of the low-latency initial trigger data transmitted through TDRSS. Instead it will be downloaded at a later time using Near Earth Network (NEN) system, which has greater bandwidth. The NEN system is available to BurstCube about once per day, and the on flight TTE buffer is approximately $48 \mathrm{hrs}$ long.

TTE data around external GRB triggers and gravitational wave events will also be downloaded. In addition, a communication channel will be provided to the community to send requests to downlink TTE data around events of interest from the last $\sim 48 \mathrm{hrs}$. While BurstCube has attitude control capabilities, it will not reorient towards specific targets. Most of the time the instrument will be pointing towards zenith, except when increased light collection by the solar panels is needed.

\section{Conclusions}

BurstCube is a 6U CubeSat to detect Gamma-Ray Bursts, scheduled to launch in 2022. By increasing the sky coverage and providing rapid alerts it will improve the probability of detecting and localizing gravitational-wave counterparts. BurstCube will also serve as a pathfinder for the next generation of wide field-of-view gamma-ray detectors in the energy range between a few tens of $\mathrm{keV}$ to a few $\mathrm{MeV}$.

\section{References}

[1] B. P. Abbott et al. Multi-messenger observations of a binary neutron star merger. The Astrophysical Journal, 848(2):L12, October 2017.

[2] A. Zoglauer, R. Andritschke, and F. Schopper. Megalib - the medium energy gamma-ray astronomy library. New Astronomy Reviews, 50(7):629-632, 2006. Astronomy with Radioactivities. $\mathrm{V}$.

[3] S. Agostinelli et al. Geant4-a simulation toolkit. Nuclear Instruments and Methods in Physics Research Section A: Accelerators, Spectrometers, Detectors and Associated Equipment, 506(3):250-303, 2003. 